\section{Sarcoma sinovial primario del riñón: una presentación inusual}

Herrera-Lomónaco $\mathrm{S},{ }^{1}$ González-Peralta AU, ${ }^{2}$ Soto-Zarate EF, ${ }^{2}$ Lambis-Ricardo $\mathrm{JA},{ }^{3}$ Robles-Pérez $\mathrm{K}^{1}$, Barreto $\mathrm{R}^{1}$

\section{Resumen}

ANTECEDENTES: el sarcoma sinovial es el cuarto tipo más común de los sarcomas. Suele localizarse en la región paraarticular de las extremidades inferiores y los tendones. Cuando aparece en el riñón es en pacientes de 15-40 años de edad, con un promedio de 38 años. Predomina en el sexo masculino y se manifiesta con una sensación de masa abdominal, hematuria y signos inespecíficos.

CASO CLÍNICO: paciente femenina de 59 años de edad que acudió a consulta por dolor en la región lumbar derecha, emesis y hematuria, acompañados de una masa palpable, dura e indolora en el cuadrante superior derecho. La tomografía computada reveló que el riñón derecho estaba aumentado de tamaño, con componentes de densidad heterogéneos. Se efectuó nefrectomía renal derecha. El diagnóstico patológico fue de sarcoma sinovial, confirmado por inmunohistoquímica. El desenlace fue fatal debido a un tumor recurrente que afectó la fosa renal derecha.

CONCLUSIONES: el sarcoma sinovial es una enfermedad rara, con especial predilección por ciertas zonas anatómicas. La localización renal es excepcional y en la bibliografía hay pocos casos reportados. Se comporta de manera agresiva, con poca respuesta al tratamiento lo que origina su gran morbilidad y mortalidad.

PALABRAS CLAVE: sinovial, sarcoma, neoplasia, tumor renal.

Rev Mex Urol. 2017 January;77(1):56-60.

\section{Primary synovial sarcoma of the kidney: An unusual presentation}

Herrera-Lomónaco $\mathrm{S},{ }^{1}$ González-Peralta $\mathrm{AU},{ }^{2}$ Soto-Zarate $\mathrm{EF},{ }^{2}$ Lambis-Ricardo $\mathrm{JA},{ }^{3}$ Robles-Pérez $\mathrm{K}^{1}$, Barreto $\mathrm{R}^{1}$

\section{Abstract}

BACKGROUND: Synovial sarcoma is an infrequent neoplasia and is the fourth most common type of sarcoma. It is often situated in the para-articular region of the lower limbs and tendons. Its renal location is more common in patients 15-40 years of age, with a mean of 38 years. It is predominant in the male sex and manifests as a sensation of abdominal mass, hematuria, and nonspecific signs.
${ }^{1}$ Departamento de Diagnóstico, Hospital Universitario del Caribe, Cartagena, Colombia.

${ }^{2}$ Facultad de Medicina, Universidad de Cartagena, Cartagena, Colombia.

${ }^{3}$ Departamento de Urología, Hospital Universitario del Caribe, Cartagena, Colombia.

Recibido: agosto 2016

Aceptado: enero 2017

Correspondencia

Dr. Jorge Lambis Ricardo

jotalambis@hotmail.com

Este artículo debe citarse como

Herrera-Lomónaco S, González-Peralta AU, SotoZarate EF, Lambis-Ricardo JA, Robles-Pérez K, Barreto R. Sarcoma sinovial primario del riñón: una presentación inusual. Rev Mex Urol. 2017 ene;77(1):56-60. 
CLINICAL CASE: A 59-year-old woman sought medical attention for pain in the right lumbar region, plus emesis and hematuria, accompanied by a palpable, hard, painless mass in the right upper quadrant. A computerized tomography scan revealed an enlarged right kidney with dense, heterogeneous components. Right nephrectomy was performed. The pathology report stated synovial sarcoma diagnosis that was confirmed by immunohistochemistry. Finally, the patient died due to a recurrent tumor involving the right renal fossa.

CONCLUSIONS: Synovial sarcoma is a rare entity with a special predilection for certain anatomic zones. Renal location is exceptional and there are presently few reported cases in the literature. It is an aggressive tumor with a poor treatment response, resulting in a high morbidity and mortality rate.

KEY WORDS: Synovial sarcoma; Neoplasia; Kidney tumor

\author{
${ }^{1}$ Departamento de Diagnóstico, Hospital \\ Universitario del Caribe, Cartagena, Co- \\ lombia. \\ ${ }^{2}$ Facultad de Medicina, Universidad de \\ Cartagena, Cartagena, Colombia. \\ ${ }^{3}$ Departamento de Urología, Hospital Uni- \\ versitario del Caribe, Cartagena, Colombia. \\ Correspondence \\ Dr. Jorge Lambis Ricardo \\ jotalambis@hotmail.com
}

\section{ANTECEDENTES}

El sarcoma sinovial es una enfermedad rara, que afecta sitios anatómicos muy particulares. En la mayor parte de los casos se afecta el tejido blando somático, y ello representa 15\% de los sarcomas en pacientes de 10 a 18 años de edad y $1.6 \%$ de los sarcomas en individuos mayores de 50 años de edad. ${ }^{1}$ Desde el punto de vista clínico y patológico el sarcoma sinovial se define como un tumor que aparece y crece en los tejidos blandos yuxtaarticulares o en las extremidades de jóvenes y adultos. ${ }^{2}$ Entre los sarcomas del riñón, el leiomiosarcoma es el tipo más frecuente, que comprende $40-60 \%$, seguido de rabdomiosarcoma, condrosarcoma, liposarcoma, angiosarcoma, hemangiopericitoma y osteosarcoma. ${ }^{2-4}$ El hallazgo de un sarcoma sinovial primario del riñón es verdaderamente excepcional y su etiología es incierta, con presentación por demás rara. Su comportamiento es agresivo, con poca respuesta al tratamiento, circunstancia que eleva la morbilidad y la mortalidad. Enseguida se expone el caso de una paciente con sarcoma sinovial primario del riñón.

\section{CASO CLÍNICO}

Paciente femenina, de 59 años de edad, con antecedentes de diabetes, obesidad, hipertensión y urolitiasis. Acudió a consulta debido a un dolor agudo en la región lumbar derecha, sin respuesta a los analgésicos, asociado con hematuria y emesis. Durante el examen físico se palpó una masa firme, circunscrita e indolora, localizada en el hipocondrio derecho. La ecografía renal reportó una masa definida, de bordes lobulados de $14 \times 11 \times 13 \mathrm{~cm}$ de extensión, con áreas hipoecoicas en su interior y flujo sanguíneo, situada en el flanco derecho. La urografía por tomografía computada evidenció al riñón derecho aumentado de tamaño y una imagen hiperdensa, de 14 mm de diámetro, con evidente dilatación ureteral ipsilateral, acompañada de una gran bolsa hidronefrótica, con componentes isodensos e hipodensos sugerentes de hidropionefrosis.

Se practicó una nefrectomía derecha; la pieza quirúrgica mostró una lesión tumoral carnosa, friable, con áreas necróticas y de sangrado que afectaban todo el espécimen (Figura 1). El examen histológico reveló un tumor maligno, con 


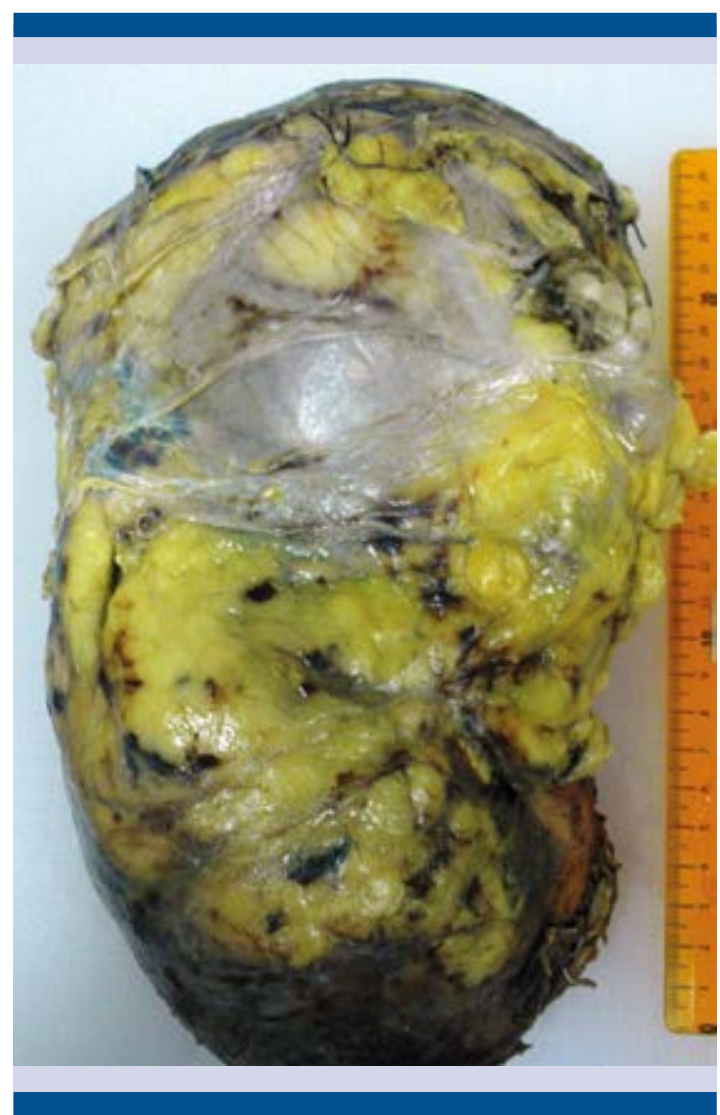

Figura 1. Pieza macroscópica donde se aprecia el aspecto carnoso con zonas de necrosis y hemorragia central.

morfología bifásica, consistente en proliferación de células fusiformes, con atipia variable, áreas de fibrosis y otras de necrosis. El examen inmunohistoquímico indicó reactividad de la muestra, con patrón de tinción difuso para $\mathrm{BCL}$ 2 y vimentina y patrón de tinción focal para P-63, CD-10 y CD-99 (Figura 2). Fue negativo para S100, miogenina, CD34 y antígeno de membrana epitelial. Estos resultados condujeron al diagnóstico de sarcoma sinovial. Tres meses después de la cirugía la paciente retornó al hospital debidoa a la reaparición del dolor abdominal y vómitos; por sus antecedentes se efectuó una urografía por tomografía computada que reportó una masa heterogénea, sugerente de recurrencia tumoral, de aproximadamente $8 \mathrm{~cm}$, ubicada en la fosa renal derecha, con afectación e infiltración en el ángulo hepático y el diafragma, sin linfadenopatías asociadas. La evolución clínica fue tórpida, con fallecimiento de la paciente durante la hospitalización.

\section{DISCUSIÓN}

Los primeros en describir el sarcoma sinovial fueron Faria y colaboradores, en 1999.5,6 El sarcoma sinovial es más frecuente en hombres, que en mujeres, con una razón hombre-mujer 1.7:1. Los límites de edad a la presentación varían de 13 a 67 años, con un promedio de 37 años, aunque también han sido descritos en la infancia. ${ }^{7}$ Lo común es que se localicen en las extremidades inferiores, a nivel de la cápsula articular, bursas y tendones pero solo $1 \%$ de los casos se localiza en el riñón. Al momento del diagnóstico, el diámetro promedio del tumor es de $11 \mathrm{~cm}$ (límites 3-21 cm). ${ }^{3}$ Por lo general, los tumores renales (independientemente de su

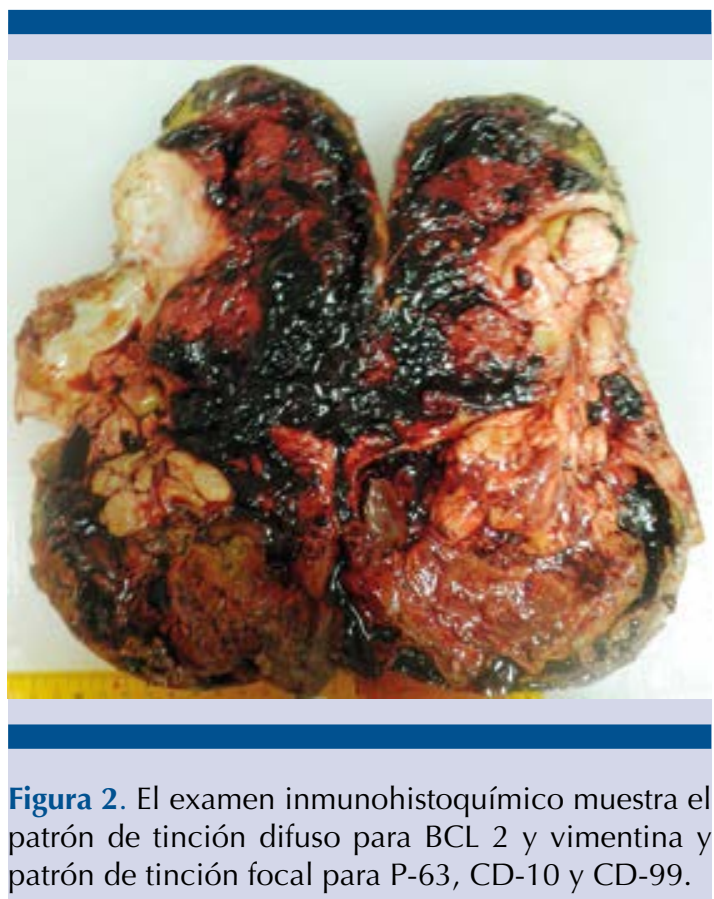


estirpe histológica) se manifiestan con la triada de dolor en la fosa lumbar o flanco, hematuria y masa palpable. Fei-X y su equipo mostraron, en un pequeño grupo de pacientes con sarcoma sinovial, los mismos síntomas: dolor de espalda baja ( $\mathrm{n}=3)$, hematuria $(\mathrm{n}=2)$, náuseas y vómitos $(n=1) .{ }^{8}$ En el caso que aquí se reporta, el sarcoma sinovial se manifestó con la triada sintomática, en el límite de edad y el promedio de tamaño descrito en la bibliografía al momento del diagnóstico.

Para el diagnóstico no existe característica clínica o imagenológica que sean determinantes ${ }^{4,6}$ sin embargo, los médicos debiéramos ser capaces de reconocer algunos patrones de imagen que hagan sospechar esta neoplasia. Un estudio protocolizado muestra algunas características generales del tumor a través de la tomografía axial computada abdominal. Ésta, sin contraste, mostró masas completas o parcialmente definidas, con parches de baja intensidad, algunas de características heterogéneas $(n=5)$ y otras homogéneas ( $n$ =1). La mayor parte de los tumores tenían la apariencia de masas sólido-quísticas o con pseudocápsulas que mostraban moderada heterogeneidad $(n=5)$ o realce del contenido $(\mathrm{n}=2)$. En ninguno de los pacientes se reportaron linfadenopatías regionales. ${ }^{8}$ Otros hallazgos ultrasonográficos son las grandes masas quísticas con o sin forma multilobulada y contenido heterogéneo, como lo muestra el caso en cuestión..$^{2,9-13}$

Desde el punto de vista histológico el sarcoma sinovial se divide en monofásico y bifásico de acuerdo con la existencia de elementos glandulares y componentes epiteliales definidos. Los sarcomas sinoviales pobremente diferenciados son una forma de evolución del tumor que puede reconocerse en ambos subtipos. ${ }^{1}$ El sarcoma sinovial pobremente diferenciado se forma a partir de láminas de células redondas indiferenciadas con núcleos hipercromáticos con una tasa mitótica alta; su pronóstico es muy pobre. ${ }^{3,12}$

Entre los aspectos inmunohistoquímicos destaca que este tumor expresa marcadores epiteliales de distribución focal, como: citoqueratina, vimentina, proteínas de CD-99, CD-56, Bcl-2, TLE1 y antígeno de membrana epitelial, ${ }^{4,10}$ pero no expresa positividad a la tinción para los marcadores: actina, desmina, S-100 y CD-34..$^{1,2}$

En el diagnóstico diferencial deben tenerse en cuenta las lesiones quísticas, ${ }^{12}$ renomegalia ${ }^{14} y$ neoplasias, como el tumor de Wilms, carcinoma de células transicionales, carcinoma de células renales, hemangiopericitoma y los tumores neuroectodérmicos primitivos. ${ }^{3,4,11} \mathrm{El}$ diagnóstico diferencial es más difícil entre pacientes con carcinoma sarcomatoide de células renales, tumores fibrosos solitarios, tumores neuroectodérmicos primitivos y otros tumores indiferenciados. A diferencia del sarcoma sinovial, el carcinoma sarcomatoide de células renales no expresa el TLE1. ${ }^{1}$ Los leiomiosarcomas y fibrosarcomas también son masas sólidas que exhiben células alargadas, parecidas a las del sarcoma sinovial, pero sin expresar marcadores BCL2, CD99 o CD56. El tumor de Wilms también muestra un patrón histológico parecido y debe tenerse en cuenta en pacientes jóvenes, aunque la fusión de los genes SYT-SSX está ausente. ${ }^{2}$

La traslocación $\mathrm{t}(\mathrm{X} ; 18)(\mathrm{p} 11.2 ; q 11.2)$ es específica para estos tumores, sin importar el grado de la diferenciación tisular y puede demostrarse en $90 \%$ de los sarcoma sinoviales. ${ }^{11,12}$

El diagnóstico definitivo se establece con la identificación de la traslocación genética, a través de la reacción en cadena de la polimerasa reversa e hibridación in situ con fluorescencia. ${ }^{2,4,6}$ En este caso en particular, el diagnóstico se estableció sólo con base en los aspectos microscópicos morfológicos y la inmunohistoquímica. 
Debido a la poca frecuencia de este tumor no existen guías de tratamiento estandarizadas, de ahí que la conducta se base en los tratamientos descritos en la bibliografía, que casi siempre incluyen a la quimioterapia y las intervenciones quirúrgicas. La quimioterapia adyuvante es con ifosfamida y doxorrubicina, previa a la nefrectomía radical; esto ha favorecido la remisión y ha mejorado la supervivencia global de algunos pacientes, incluso con tumores poco diferenciados con metástasis. ${ }^{13}$ A pesar del tratamiento, el pronóstico no es claro debido al limitado número de casos. ${ }^{2}$

\section{CONCLUSIÓN}

El sarcoma sinovial es una entidad rara, con especial predilección por ciertas zonas anatómicas. La localización renal es excepcional y hasta la fecha existen pocos casos reportados en la bibliografía. Se comporta de manera agresiva, con poca respuesta al tratamiento lo que incrementa la morbilidad y mortalidad.

\section{Conflicto de intereses}

Ninguno por declarar

\section{REFERENCIAS}

1. Schoolmeester JK, Cheville JC, Folpe AL. Synovial sarcoma of the kidney: a clinicopathologic, immunohistochemical, and molecular genetic study of 16 cases. Am J Surg Pathol. 2014;38(1):60-5. Epub 2013/09/26.

2. Ozkan EE, Mertsoylu H, Ozardali HI. A case of renal synovial sarcoma treated with adjuvant ifosfamide and doxorubicin. Intern Med. 2011;50(15):1575-80. Epub 2011/08/02.
3. Wang Z, Zhong Z, Zhu L, et al. Primary synovial sarcoma of the kidney: A case report. Oncol Lett. 2015;10(6):3542-4. Epub 2016/01/21.

4. Schaal $\mathrm{CH}$, Navarro FC, Moraes Neto FA. Primary renal sarcoma with morphologic and immunohistochemical aspects compatible with synovial sarcoma. Int Braz J Urol. 2004;30(3):210-3. Epub 2005/02/04.

5. Faria P, Argani P, Epstein J, et al. Primary synovial sarcoma of the kidney: a molecular reappraisal of a subset of socalled embryonal renal sarcoma. Lab Invest. 1999;79(94A).

6. Argani P, Faria PA, Epstein JI, et al. Primary renal synovial sarcoma: molecular and morphologic delineation of an entity previously included among embryonal sarcomas of the kidney. Am J Surg Pathol. 2000;24(8):1087-96. Epub 2000/08/10.

7. Radhakrishnan V, Dhanushkodi M, Narayanswamy K, et al. Synovial sarcoma of kidney in a child: A rare presentation. J Indian Assoc Pediatr Surg. 2016;21(2):75-7. Epub 2016/04/06.

8. Lv XF, Qiu YW, Han LJ, et al. Primary renal synovial sarcoma: computed tomography imaging findings. Acta Radiol. 2015;56(4):493-9. Epub 2014/04/01.

9. Mishra S, Awasthi N, Hazra SP, et al. Primary synovial sarcoma of the kidney. Saudi J Kidney Dis Transpl. 2015;26(5):996-9. Epub 2015/09/12.

10. Hirose $\mathrm{M}$, Mizuno $\mathrm{K}$, Kamisawa $\mathrm{H}$, et al. Clear cell sarcoma of the kidney distinguished from synovial sarcoma using genetic analysis: a case report. BMC Res Notes. 2015;8:129. Epub 2015/04/19.

11. Kohle O, Abt D, Rothermundt C, et al. Soft tissue sarcomas of the kidney. Rare tumors. 2015;7(1):5635. Epub 2015/04/29.

12. Nishida $\mathrm{T}$, Inamoto $\mathrm{T}$, Uehara $\mathrm{H}$, et al. Monophasic primary renal synovial sarcoma accompanied with a hemorrhagic cyst. Urol J. 2011;8(3):244-7. Epub 2011/09/13.

13. Park SJ, Kim HK, Kim CK, et al. A case of renal synovial sarcoma: complete remission was induced by chemotherapy with doxorubicin and ifosfamide. Korean J Intern Med. 2004;19(1):62-5. Epub 2004/04/01.

14. Modi $G$, Madabhavi I, Panchal $H$, et al. Primary synovial sarcoma of kidney: a rare differential diagnosis of renomegaly. Case Rep Pathol. 2014;2014:657497. Epub 2015/01/23. 